\title{
Condition Monitoring of Motorised Devices for Smart Infrastructure Capabilities
}

\author{
Pritesh Mistry $(\bowtie)$, Phil Lane, Paul Allen, Hussain Al-Aqrabi, and Richard Hill \\ School of Computing and Engineering, University of Huddersfield, UK \\ \{p.mistry, p.lane, p.d.allen, h.al-aqrabi, r.hill\}@hud.ac.uk
}

\begin{abstract}
This paper presents a signal processing methodology based on fast Fourier transform for the early fault detection of electrically motorised devices. We used time-stamped, current draw data provided by Network Rail, UK, to develop a methodology that may identify imminent faults in point machine operations. In this paper we describe the data, preprocessing steps and methodology developed that can be used with similar motorised devices as a means of identifying potential fault occurrences. The novelty of our method is that it does not rely on labelled data for fault detection. This method could be integrated into smart city infrastructure and deployed to provide automated asset maintenance management capabilities.
\end{abstract}

Keywords: Condition monitoring - Fault detection · Point machines . Fast Fourier transform - Smart city.

\section{Introduction}

Smart cities are urban areas that implement electronic sensors and detection devices to collect and process data. These physical devices make up the internet of things (IoT) and aim to provide a means to manage services, assets, and resources within a city $[4,10,8]$. Cities are complex systems, the idea of a smart city emerged as a means to facilitate the communication between information and communication technologies (ICT), that would be advantageous to serve a citys needs. Indeed a city can only be considered "smart" if it is able to synthesis these data in some intelligent manner.

Harrison et al [6] described smart cities to have three key components, They must be instrumented, to capture real-world live data through the use of sensors and devices. Interconnected, to allow the integration of collected data into computing platforms, and intelligent, to explore, model, and analyse the data to leverage the information contained within, for the benefit of the city.

A railway infrastructure could be considered smart if it is able to employ smart technology to provide autonomy to its functionalities. One aspect of such 
autonomy could be the detection of fault occurrences. Such capabilities are desirable for many reasons, primarily from a safety perspective [13], but also to streamline servicing and repairs to offer minimal disruption to passengers.

Point machines are a critical part of a railway infrastructure, used for switching and locking railway turnouts. Point machines consist of an electrically driven rotary motor, whose movement is transmitted through gearing to produce linear movement to lock or unlock the rails [12] changing their position from normal (NR) to reverse $(\mathrm{RN})$ and vice versa. The use of an electric motor in point machines makes current based fault identification viable. A point machine will undergo a process of degradation as normal operation tends to complete failure. If the progression or rate of degradation can be identified over time then maintenance can be arranged prior to failure occurrence.

Although guidelines for point machine operation and replacement exist, such as, replacement after a fixed time period or after a number of accumulated actuations, these guidelines do not take into consideration the operational and environmental conditions such as the track load or weather. As such, faults may occur unexpectedly, so more robust fault identification methods are required. When considering fault detections for point machine failures, the early approaches relied on threshold settings, but produced limited success with a high proportion of false alarms [9]. More recent methods used historical data and machine learning techniques $[2,1]$ to identify anomalies that could signify machine wear and potential imminent failure.

Asada et al [1] extracted features of point machine electrical power consumption using discrete wavelet transform and trained a support vector machine (SVM) to produce predictive accuracies of $100 \%$. Similarly Bian et al [3], used self-organising maps (SOM) and SVM to identify degradation states of point machines before classifying the degradation states to $96.97 \%$ accuracies. An interesting approach by Garcia et al [5] used "expected" current signals from historical data and compared these to measured signals. A VARMA (vector autoregressive moving-average) model and harmonic regression model were used to generate the "expected" signal forms. If the incoming signal produced a trace beyond the "expected" model a fault was deemed to have been detected.

\section{Data}

The data used for this study were supplied by Network Rail, UK, and covered three regions; London North Eastern (LNE), London North Western (LNWN) and Sussex. The data were collected over the months of April 2018 to June 2018, with each region containing several thousand csv (comma-separated values) files. Each file is a daily log for every actuation of a point machine in a given direction (either NR or RN), recorded in a continuous sequential manner for a given day. Each file is labelled with the point machine name, date of logging, and direction 
of movement (NR or RN). Within each file, the current draw in amperes (amps or A) is recorded against a millisecond time-stamp for every actuation that occurred on a given day. Table 1 shows the number of files and point machines present in each of the three regions. A total of 45,690 files were initially available in the dataset. All the data available are unlabelled data and therefore no indication of fault occurrences are known.

Table 1. Number of point machine instruments across each region, and files present in the dataset

\begin{tabular}{|c|c|c|}
\hline Region & No. of files & No. of point machines \\
\hline \hline LNE & 15,802 & 103 \\
LNWN & 17,081 & 129 \\
SUSSEX & 12,807 & 99 \\
\hline
\end{tabular}

An example of a typical file containing current draw time-stamped data is shown in Table 2. The table shows the first five records (Row0-Row4) and the last five records (Row242-Row246) of the first actuation for point machine ALTOFTJT_NOC_P2443B, in the NR direction logged on the 1st June 2018. The table also shows the first five records (Row247-Row251) of the second actuation of the same point machine in the NR direction on the same day. The frequency of data logs during actuation is approximately 10 milliseconds. Once an actuation is complete the data log stops until the next actuation is actioned. An example of this can be seen between Row246 and Row247 of Table 2, which shows the end of one actuation log and the beginning of another. The time-stamp for these records moves from 2018-06-01 02:28:07.977 to 2018-06-01 05:30:57.793, which is a time difference of many minutes, suggesting the two entries cannot be for the same actuation event. It is also worthwhile to note that any given actuation begins with a current reading of $0.00 \mathrm{~A}$ (zero amps) and ends with a current reading of $0.00 \mathrm{~A}$, as can be seen for the records at Row0 (start of first actuation) and Row246 (end of first actuation) and again at Row247 (start of second actuation).

\subsection{Data Preprocessing}

Real-world data is seldom error free and as such an element of preprocessing and data cleansing was required. For this study all the data preprocessing and analysis was performed using the Knime analytics platform [7] and R Statistical package [11]. In the first instance, all 45,690 files (LNE, LNWN and Sussex) were checked for errors. Any files that contained missing records or erroneous values were identified and removed from the data. It also transpired that the data contained files with duplicate names. Unable to ascertain the validity of these files, 
Table 2. Current draw for point machine ALTOFTJT_NOC_P2443B, of direction NR, logged on the 1st June 2018.

\begin{tabular}{|c|c|c|}
\hline Row No & DateTime & ALTOFTJT_NOC_P2443B_NR_current \\
\hline \hline Row0 & 2018-06-01 02:28:05.527 & 0.0 \\
\hline Row1 & 2018-06-01 02:28:05.537 & 13.18 \\
\hline Row2 & 2018-06-01 02:28:05.547 & 19.43 \\
\hline Row3 & $2018-06-0102: 28: 05.557$ & 18.04 \\
\hline Row4 & $2018-06-0102: 28: 05.567$ & 16.15 \\
\hline$\vdots$ & $\vdots$ & $\vdots$ \\
\hline Row242 & $2018-06-0102: 28: 07.937$ & 0.82 \\
\hline Row243 & $2018-06-0102: 28: 07.947$ & 0.81 \\
\hline Row244 & $2018-06-0102: 28: 07.957$ & 0.80 \\
\hline Row245 & $2018-06-0102: 28: 07.967$ & 0.76 \\
\hline Row246 & $2018-06-0102: 28: 07.977$ & 0.00 \\
\hline Row247 & $2018-06-0105: 30: 57.793$ & 0.00 \\
\hline Row248 & $2018-06-0105: 30: 57.803$ & 0.81 \\
\hline Row249 & $2018-06-0105: 30: 57.813$ & 16.22 \\
\hline Row250 & $2018-06-0105: 30: 57.823$ & 19.37 \\
\hline Row251 & $2018-06-0105: 30: 57.833$ & 17.55 \\
\hline$\vdots$ & $\vdots$ & $\vdots$ \\
\hline & & \\
\hline
\end{tabular}

all duplicates were removed from the data. After this initial preprocessing step the number of remaining files and point machines available for analysis are shown in Table 3. A total of 23,389 files remained. In this study the normal (NR) and reserve $(\mathrm{RN})$ profiles were not considered separately. Rather, the presumption was taken that should a fault occur with the operation of an instrument that is identifiable from the current draw, then this fault would be observable in both the normal (NR) and reverse (RN) direction.

Table 3. Number of point machine instruments across each region after preprocessing

\begin{tabular}{|c|c|c|}
\hline Region & No. of files & No. of point machines \\
\hline \hline LNE & 6,533 & 92 \\
LNWN & 9,715 & 105 \\
SUSSEX & 7,141 & 91 \\
\hline
\end{tabular}




\section{Methodology}

For fast Fourier transform (FFT) analysis it was necessary to separate each actuation of every point machine in the data which remained. Since the data is presented in a continuous sequential manner, to separate individual actuations, the time difference between recorded instances was calculated within a file. Where a difference of $\geq 500$ milliseconds and a $0.00 \mathrm{~A}$ current reading between adjacent records existed, this indicated the beginning of a new actuation event. For example, in Table 2 the time difference between Row246 and Row247 is $>500$ milliseconds with a $0.00 \mathrm{~A}$ current reading, this therefore indicates the beginning of a new actuation event for that point machine. Conversely, the time difference between Row247 and Row248 is $<500$ milliseconds and therefore indicates those records are of the same actuation event. An example of the current draw signal in the normal (NR) and reverse $(\mathrm{RN})$ direction for point machine ALTOFTJT_NOC_P2443B is shown in Fig 1.

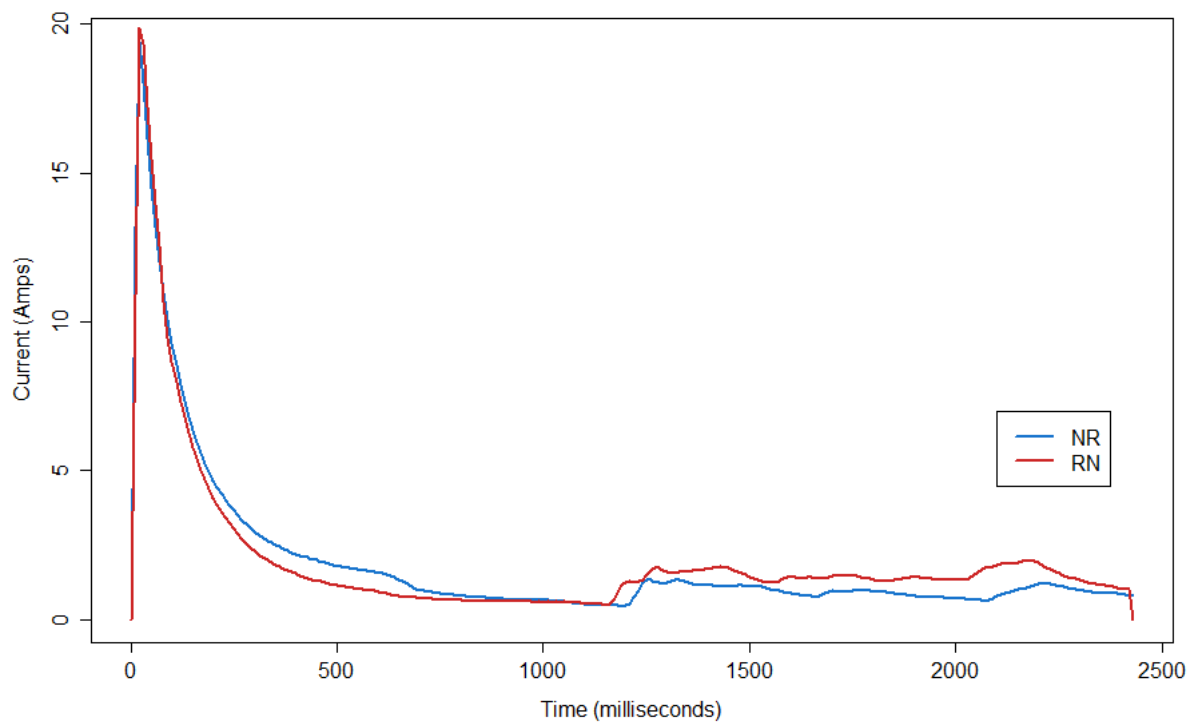

Fig. 1. Current draw profile of point machine ALTOFTJT_NOC_P2443B actuations in the normal $(\mathrm{NR})$ and reverse $(\mathrm{RN})$ directions.

\subsection{Fast Fourier Transform (FFT)}

The signal produced by each actuation of a point machine is represented by the current (amps) changing over time as shown in Fig 1. This is commonly referred 
to as the time domain. A fast Fourier transform can be applied to deconstruct a time domain signal into a frequency domain representation. These frequency domain components are represented as discrete values or bins and used to analyse the different frequencies present in the original signal.

For each point machine, individual actuation profiles were separated as described above. Typically each profile resulted in approximately 250 instances of data. As such, a zero padding was added to the end of each profile that made all profiles up to 512 samples. A sliding window of length 512 and step size of 256 was used for the input signal. To prevent spectral leakage and enhance the ability of an FFT to extract spectral data the Hann windowing function was used. The goal of minimising leakage is to prevent an FFT from producing the wrong frequencies. When an FFT is used to measure the frequency component it assumes a finite data set, or simply, a single period of a periodic signal. When the signal data used is not periodic, windowing helps to reduce the amplitude of the discontinuous data at the boundaries. A frequency domain plot of the data in Table 2 is shown in Fig 2.

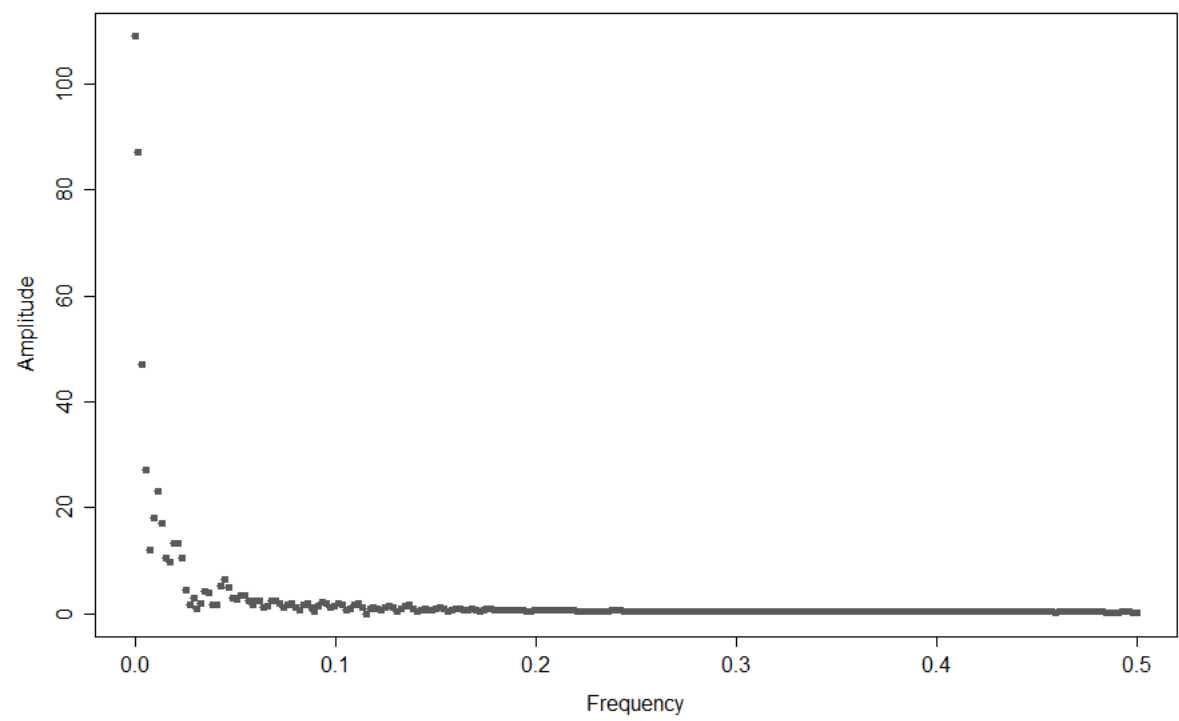

Fig. 2. Frequency domain plot following FFT of the signal data from the actuation of point machine ALTOFTJT_NOC_P2443B in the normal (NR) direction. 


\subsection{Curve fitting}

To identify anomalies in the processed signal, an attempt was made to fit a curve to the frequency domain data shown in Fig 2. The goodness of fit achieved from second and third order polynomials were not satisfactory. Therefore it was decided to take the $\log _{10}$ value of both the frequency and amplitude values of the FFT. Using the $\log _{10}$ values resulted in a more linear relationship of the frequency domain and amplitude. A linear curve could then be fitted to the data and the goodness of fit calculated. A plot of the $\log _{10}$ values and fitted curve is shown in Fig 3. A linear curve was fitted using the R statistical package [11], with the residual standard error obtained for further analysis.

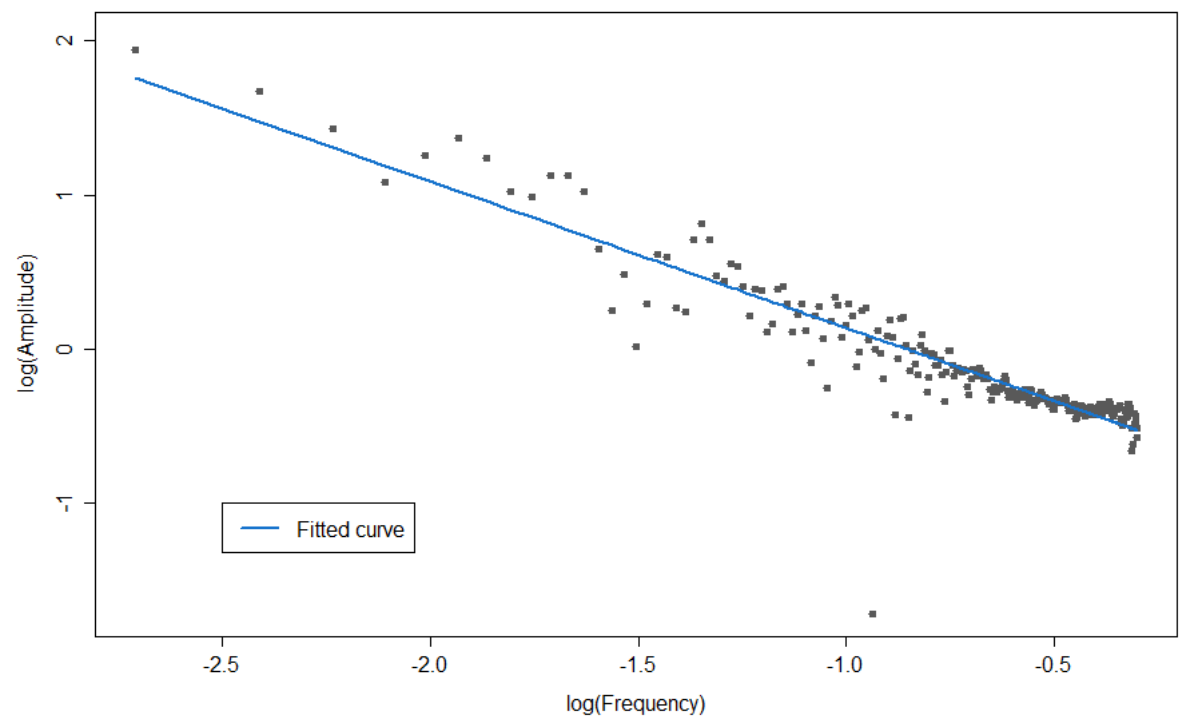

Fig. 3. Linear curve fitting to log values of FFT analysis.

\section{Results}

The methodology proposed is based on the premise that a poorly functioning point machine can be identified from the signal data it produces. A point machine that produces an irregular unexpected signal is more likely to be showing signs of wear and imminent failure. The process of FFT and curve fitting to ascertain goodness of fit is a novel approach for fault detection. Although the data used in this study is unlabelled, and therefore cannot unequivocally be assigned to a fault, we can gauge how well this methodology works from the residual standard 
error values produced. For actuations where a fault is not apparent we expect the residual standard error to be comparatively lower.

Fig 4 shows the time domain signal of point machine ALTOFTJT_NOC _P2443A when moving in the reverse direction (RN), whilst Fig 5 shows a linear curve fitted to the log of the frequency and amplitude upon FFT analysis of the same actuation. From the data processed in this study the signal produced in Fig 4 seems typical of a point machine in good working order. This is further supported by the residual standard error of the fitted curve shown in Fig 5 which produced a value of 0.064 .

Compare this with the time domain signal of point machine ALTOFTS_TJC3 _P2442A in the reverse direction (RN) as shown in Fig 6. It is immediately apparent from the signal profile that the period from approximately $500-1500$ milliseconds is not as smooth as that of the same period in Fig 4. Furthermore the $\log$ of the frequency and amplitude upon FFT analysis shown in Fig 7 produces a greater residual standard error value of 0.256 .

Using the residual standard error values of every actuation of a given point machine, a profile of the operational characteristics of an instrument can be produced. Plotting the residual standard errors over time quickly shows where an instrument has potentially failed or about to fail. Fig 8 shows a residual standard error plot for all the actuations of point machine APPLYJCT_TJC3_P5109 in the normal (NR) direction. Over 1250 actuations are shown, all of which produce a very consistent baseline residual standard error value of approximately 0.05 .

Similarly Fig 9 shows a residual standard error plot for all the actuations of point machine ALTOFTJT_NOC_P2443A in the normal (NR) direction. On this plot however, the baseline residual standard error value, of approximately 0.10, is higher than for that of point machine APPLYJCT_TJC3_P5109 (Fig 8). Furthermore there is one actuation event which produces a residual standard error value of $>0.30$, much higher than the baseline for the instrument, which may suggest operational issues with the instrument. Other actuations along the time line have produced residual standard error values that begin to push beyond the baseline value and may signify the development of a fault. 


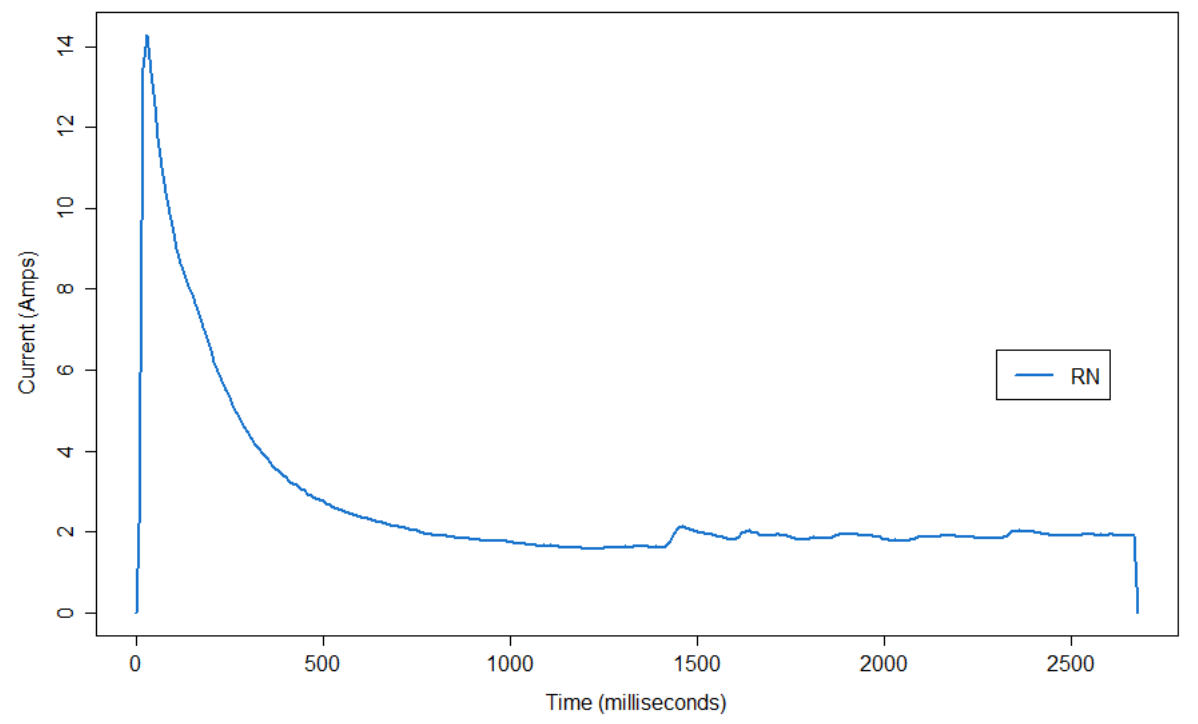

Fig. 4. Time domain signal of point machine ALTOFTJT_NOC_P2443A actuation in the reverse $(\mathrm{RN})$ direction.

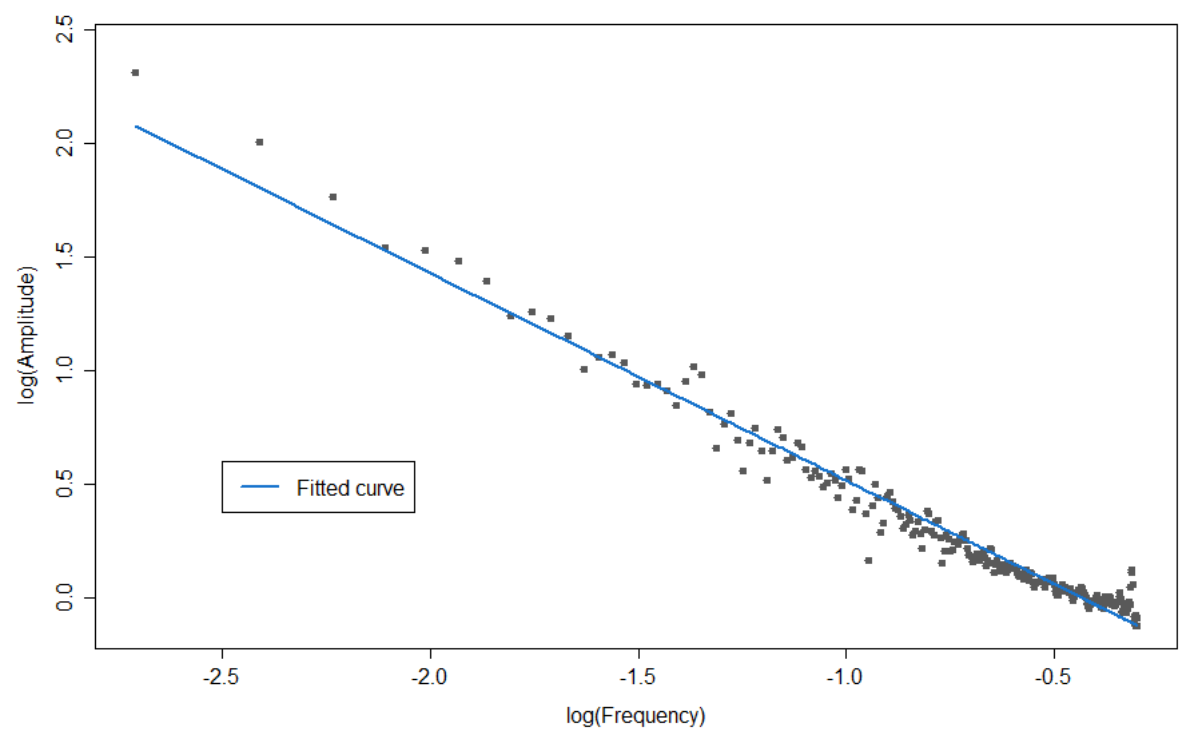

Fig. 5. Curve fitting of the FFT analysis of point machine ALTOFTJT_NOC_P2443A shown in Fig 4. Residual standard error $=0.064$. 


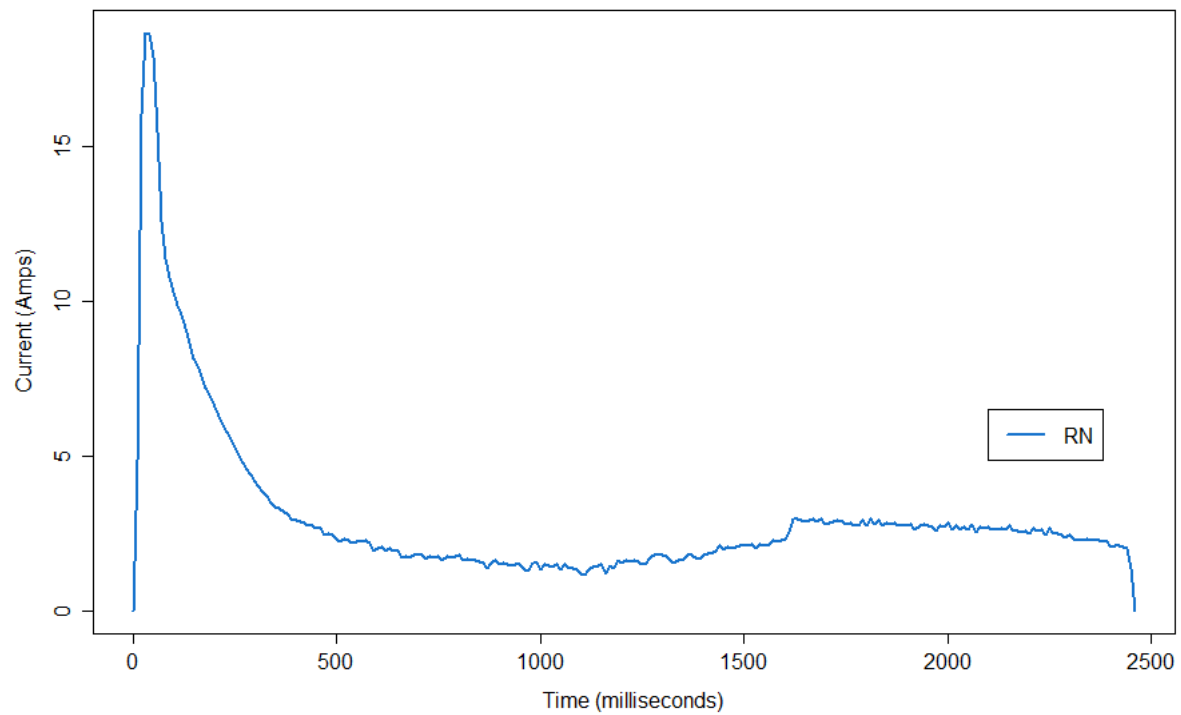

Fig. 6. Time domain signal of point machine ALTOFTS_TJC3_P2442A actuation in the reverse $(\mathrm{RN})$ direction.

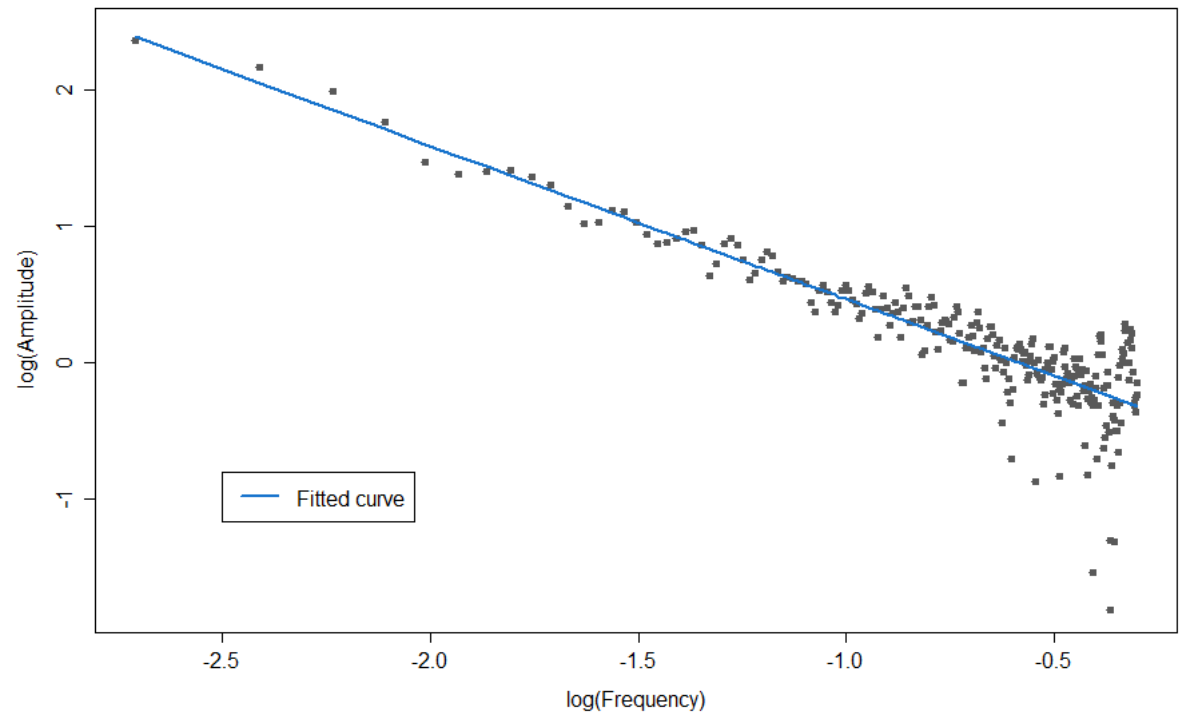

Fig. 7. Curve fitting of the FFT analysis of point machine ALTOFTS_TJC3_P2442A shown in Fig 6. Residual standard error $=0.256$ 


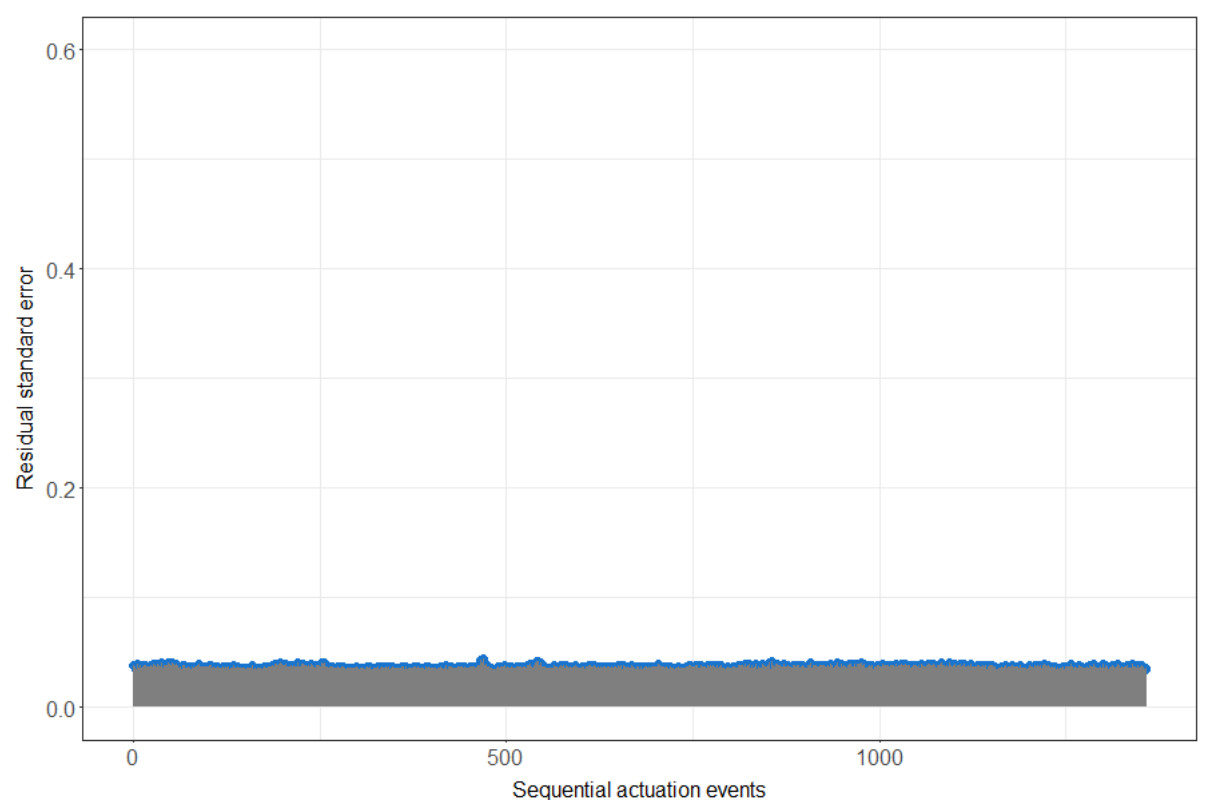

Fig. 8. Residual standard error of sequential actuation events for point machine APPLYJCT_TJC3_P5109 in the NR direction.

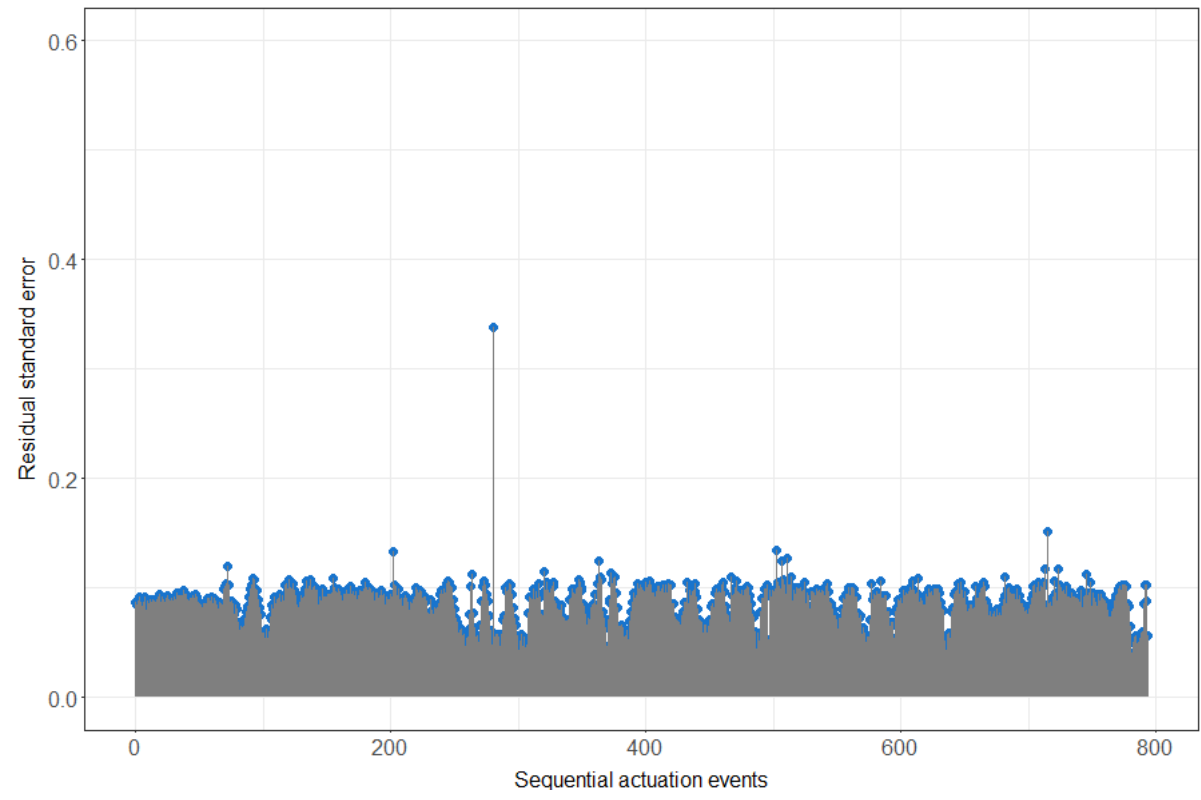

Fig. 9. Residual standard error of sequential actuation events for point machine ALTOFTJT_NOC_P2443A in the NR direction. 


\section{Conclusion}

In this study we use point machine data supplied by Network Rail, UK, to propose a novel methodology for the condition monitoring of motorised devices. Using time domain signal data of instruments fitted with current sensing capabilities together with FFT analysis, we have shown how frequency domain data can be interpreted to build a fault diagnosis capability which can be implemented into smart city technology.

Many studies are published in the literature which show fault detection methods using experimental or simulated data and machine learning techniques to classify faults from pre-labelled data. The novelty of our work is that we use a large volume of real-world unlabelled data. As such we do not know from the data when a fault occurs, which mimics the real world more accurately. It is likely from the information we have presented that there are observable differences in the signal profiles of good and impaired actuations. Our methodology exploits these differences and puts forward a method of condition monitoring for individual instruments over time. The proposed methodology is not directly comparable to other studies that have developed fault classification models. Rather we have proposed a methodology for which faults may be observable from unlabelled real-world data with the potential of real time analysis.

As seen from the evidence presented in our results, the behavioural characteristics of each point machine is unique to that machine. The advantage of this methodology is that it is dynamic to individual instruments and does not rely on a "one size fits all" thresholding approach. Rather it is able to treat each instrument separately and therefore monitor at the individual level.

Our future efforts will focus on using machine learning techniques to establish dynamic levels of residual standard error values for different point machines. It is evident from the data that point machines do not operate at equal baselines. Therefore, developing a system which can establish limits by learning the behaviour unique to each point machine would likely help reduce false positive

detections. Furthermore it may be beneficial to separate the normal (NR) and reverse (RN) directions of actuations for improved fault detection. 


\section{References}

[1] T. Asada, C. Roberts, and T. Koseki". "An algorithm for improved performance of railway condition monitoring equipment: Alternating-current point machine case study". In: Transportation Research Part C: Emerging Technologies 30 (2013), pp. 81-92.

[2] Chong Bian et al. "Degradation Detection Method for Railway Point Machines". In: arXiv preprint arXiv:1809.02349 (2018).

[3] Chong Bian et al. "Degradation Detection Method for Railway Point Machines". In: (Sept. 2018).

[4] T. M. Chen. "Smart grids, smart cities need better networks [Editor's Note]". In: IEEE Network 24.2 (Mar. 2010), pp. 2-3. ISSN: 0890-8044. DOI: $10.1109 /$ MNET . 2010.5430136.

[5] Fausto P. Garcia, Diego J. Pedregal, and Clive Roberts. "Time series methods applied to failure prediction and detection". In: Reliability Engineering — 63 System Safety 95.6 (2010), pp. 698-703. ISSN: 0951-8320.

[6] C. Harrison et al. "Foundations for Smarter Cities". In: IBM Journal of Research and Development 54.4 (July 2010), pp. 1-16. ISSN: 0018-8646. DOI: $10.1147 / J R D .2010 .2048257$.

[7] Knime. Knime. https : //www . knime.com/. 2018 (accessed August 2, 2018).

[8] Juan Manuel Barrionuevo, Pascual Berrone, and Joan Ricart. "Smart Cities, Sustainable Progress: Opportunities for Urban Development". In: IESE Insight (Sept. 2012), pp. 50-57. DOI: 10.15581/002 . ART-2152.

[9] F P Garcia Marquez, C Roberts, and A M Tobias. "Railway point mechanisms: Condition monitoring and fault detection". In: Proceedings of the Institution of Mechanical Engineers, Part F: Journal of Rail and Rapid Transit 224.1 (2010), pp. 35-44.

[10] Lluisa Marsal, Joan Colomer, and Joaquim Melendez. "Lessons in urban monitoring taken from sustainable and livable cities to better address the Smart Cities initiative". In: Technological Forecasting and Social Change 90 (Feb. 2014), pp. 611-622. DOI: 10.1016/j.techfore.2014.01.012.

[11] R. R. https://www.r-project.org/. 2018 (accessed August 2, 2018).

[12] Jaewon Sa et al. "Replacement condition detection of railway point machines using an electric current sensor". In: Sensors 17.2 (2017), p. 263.

[13] Marius Vileiniskis, Rasa Remenyte-Prescott, and Dovile Rama. "A fault detection method for railway point systems". In: Proceedings of the Institution of Mechanical Engineers, Part F: Journal of Rail and Rapid Transit 230.3 (2016), pp. 852-865. DOI: 10.1177/0954409714567487. URL: https : //doi.org/10.1177/0954409714567487. 